\title{
Monitoring crack propagation in skin-stringer elements using carbon nanotube doped adhesive films: Influence of defects and manufacturing process
}

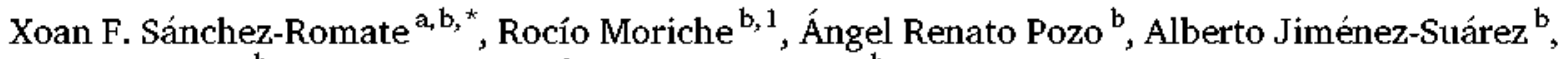 \\ María Sánchez ${ }^{b}$, Alfredo Gïiemes ${ }^{a}$, Alejandro Ureña ${ }^{b}$ \\ ${ }^{a}$ Department of Aerospace Materials and Processes, Escueda Técnica Superior de ingenieros Aeronáuticos, Universidad Politécnica de Madrid, Plaza del Cardenal Cisneros \\ 3, 28040, Modrid, Spain \\ - Materiats Science and Engineering Area, Escueta Superior de Ciencias Experimentales y Tecrología, Universidad Rey Juan Carlos, Calte Tutipân s/n, 28933, Móstotes, \\ Madrid, Spain
}

\begin{abstract}
A B S T R A C T
Standard Mode-l and skin-stringer sub-elements were manufactured using novel adhesive films reinforced with carbon nanotubes. Peeling tests were conducted to analyse the different crack propagation mechanisns. In this context, the influence of manufacturing methods and artificial defects is deeply explored. It was observed that the electrical resistance increased with crack length due to a breakage of electrical pathways, depending on manufacturing and induced defects. Co-bonded specimens showed a mote stable behaviour due to a better interface between the adhesive and substrate than joints manufactured by secondary bonding. Moreover, by analysing the influence of artificial defects, it was observed that larger discontinuities induced more unstable electromechanical behaviours as there is a more prevalent breakage of electrical pathways. In this regard, samples with Teflon inserts showed sharper increases of electrical tesistance than those previously treated with a liquid agent simulating a kissing bond. Therefore, the proposed technique shows a high potential and applicability for Structural Health Monitoring (SHM) of integrated composite structures.
\end{abstract}

\section{Introduction}

Carbon Fibre Reinforced Composites (CFRC) are being widely used in the aircraft industry due to the enhancement of specific mechanical properties in comparison to some conventional alloys. Other factors, such as their good corrosion behaviour, are also valuable and imply a great effort in developing more complex CFRC structures.

This increasing complexity leads to the development of proper joining techniques. In this regard, adhesive joints are very interesting over bolted connections due to a higher uniformity of stress distribution and weight saving [1].

However, they present some limitations such as the need of a very careful surface treatment of the substrates and their difficulty in being inspected, two crucial factors that are the subject of high interest nowadays.
On the one hand, it has been widely investigated that surface treatment drastically affects the mechanical performance of the adhesive joint $[2,3]$. In addition to that fact, the presence of defects such as interface debonding has a strong influence on the shear properties of bonded joints $[4,5]$.

On the other hand, inspection techniques such as Non-Destructive Testing (NDT) by conventional methods such as embedded Fibre Bragg Grating sensors (FBGs) or ultrasonic waves [6-8] ate often quite difficult to analyse due to their complex physical basis $[9,10]$, not giving an overview of the mechanical health of the structure. Therefore, it is necessary to explore novel NDT techniques [11]

In this regard, carbon nanoparticles, such as carbon nanotubes (CNTs) seem to be a promising alternative due to their exceptional mechanical and electrical properties [12,13] and the fact that they improve the electrical properties of the polymer matrix making them 
Table 1

Cure cycle parameters of secondary bonding.

\begin{tabular}{lll}
\hline Parameter & First Stage & Second stage \\
\hline Pressure & Ramp from 0 to $0.6 \mathrm{MPa}$ during $15 \mathrm{~min}$ & $0.6 \mathrm{MPa}$ during $90 \mathrm{~min}$ \\
Temperature & Ramp from 25 to $175^{\circ} \mathrm{C}$ during $45 \mathrm{~min}$ & $175^{\circ} \mathrm{C}$ during $60 \mathrm{~min}$ \\
\hline
\end{tabular}

electrically conductive by the creation of electrical percolating networks inside the polymer [14-16]. This electrical network makes possible their use as strain and damage sensors for Structural Health Monitoring (SHM) applications by means of electrical conductivity measurements [17-20]. More specifically, they have demonstrated their potential in flexible strain-sensors with gauge factors, that is, the change of the normalised resistance divided by the applied strain, up to 350 [21,22], as well as to detect delamination in interleaved buckypapers [23]. In this context, their applicability to crack propagation monitoring in bonded joints at quasi-static $[24,25]$ and fatigue $[26,27]$ conditions has been widely explored and showed promising results.

Furthermore, their addition generally enhances the mechanical performance of bonded joints due to the stiffening and crack-bridging effect of CNTs inside the polymer matrix [28-30].

In previous studies, a novel CNT doped adhesive film was proposed and its mechanical and sensing performance were deeply investigated. More specifically, it demonstrated an excellent capacity to properly monitor crack propagation in Single Lap Shear (SLS), Mode-I and ModeII standard tests [31-34].

Therefore, this work aims to explore the SHM capabilities of these novel CNT doped adhesive films in the crack propagation monitoring of more complex structures, such as skin-stringer sub-elements. More specifically, the effect of manufacturing techniques is evaluated, as well as the presence of artificial defects. To achieve this purpose, stiffened panels were manufactured by two techniques: co-bonding and secondary bonding. Furthermore, the effect of various types of artificial defects produced by applying a release liquid agent or Teflon inserts along the bonded joint, are evaluated. Then, a comparison between the mechanical and electrical performance of these damaged sub-elements is analysed as well as a microstructural characterisation of the joint is carried out. This would enable a more complete overview about the SHM potential of the proposed technique to be obtained.

\section{Experimental procedure}

\subsection{Adhesive preparation}

The adhesive was a $F M 300 \mathrm{~K}$ film supplied by Cytec. It is used for metal and CFRP bonding with a good thickness control, due to a knit tricot carrier.

Multi-wall Carbon Nanotubes (MWCNTs) are NC700O supplied by Nanocyl. They have an average diameter of $10 \mathrm{~nm}$ and a length up to 1.5 $\mu \mathrm{m}$ with a $95 \%$ purity.

CNT dispersion was achieved by means of ultrasonication during 20 min in an aqueous media by the help of a Sodium-Dodecyl-Sulphate (SDS) surfactant. Then, it was sprayed on the adhesive surface using an airbrush at 1 bar pressure and dried prior to curing. Weight contents were 0.1 and $0.25 \mathrm{wt} \%$ for CNTs and SDS, respectively. All the parameters were previously optimised [33].

\subsection{Joint manufacturing}

Two types of specimens were fabricated: a skin-stringer element (pristine and with induced artificial defects), and a damaged Mode-I standard coupon in order to firstly assess the influence of defects on the electromechanical behaviour of the bonded joint.

The damaged Mode-I coupon with Teflon inserts, was manufactured in a hot press with the curing cycle parameters shown in Table 1 . The schematics of the artificial defects are shown in Fig. 1 (a). The

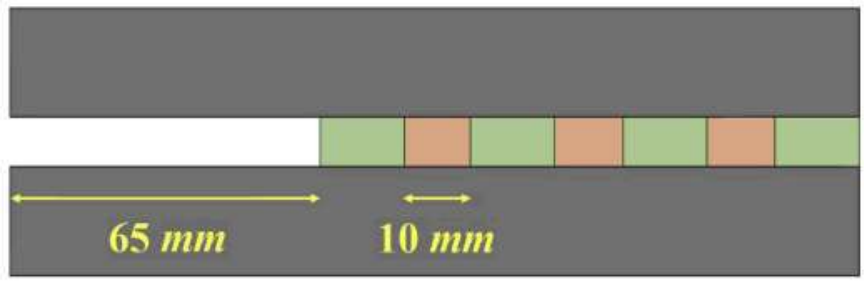

(a)

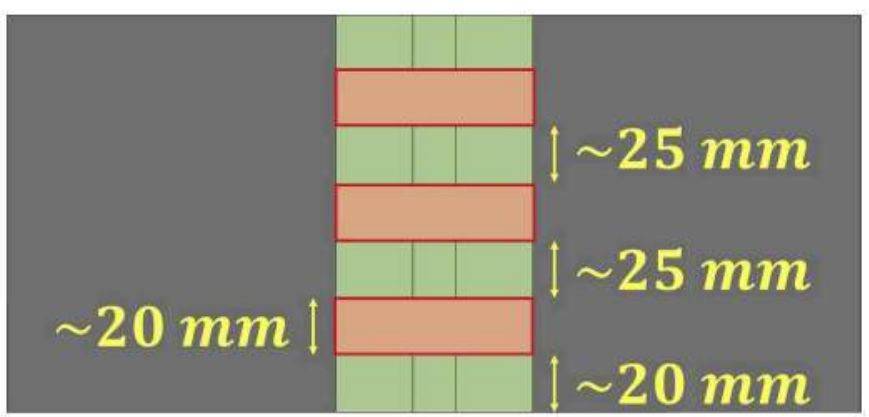

(b)

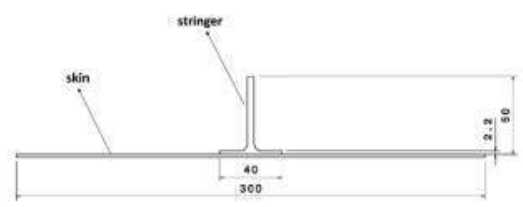

(c)

Fig. 1. Schematics of artificial defects in (a) standard Mode-I coupons and (b) skin-stringer elements where red areas indicate the presence of these discontinuities and (c) dimensions (in $\mathrm{mm}$ ) of skin-stringer elements. (For interpretation of the references to colour in this figure legend, the reader is referred to the Web version of this article.)

dimensions of the substrates were $150 \times 25 \times 3.5 \mathrm{~mm}^{3}$.

Stiffened elements were manufactured by two different bonding techniques: co-bonding of an un-cured skin with a pre-cured stringer and by secondary bonding.

Co-bonding was done by a manual lay-up process. The material used for the skin was a $2 \times 2$ twill CFRP fabric Z19723-ABS5003P-HTA (926) with a layer sequence of $[+45 / 0]_{s}$ The bonding surface of the pre-cured $\mathrm{T}$ stringer was carefully sanded and cleaned with isopropyl. Then the doped adhesive layer was manually lap-up over the surface. Then, the Tstringer was positioned along the flat uncured laminate and a vacuum bag process was performed under vacuum conditions. In order to cobond the elements, the vacuum bag was put inside an oven with a standard curing cycle using a heating ramp of $2{ }^{\circ} \mathrm{C} / \mathrm{min}$ and a stabilisation step at $180^{\circ} \mathrm{C}$ for a duration of $150 \mathrm{~min}$.

Secondary bonding was done by using a vacuum bag with a curing cycle at the same temperature as the standard coupons and UD substrates. The layer sequence used for the skin was the same than the one used for co-bonded specimens. Pristine and damaged specimens were fabricated by inducing two types of artificial defects inside the adhesive joint: on the one hand, a sample with a local inclusion of a liquid demoulding agent, Frekote, which could act as a kissing bond, and, on the other hand, a sample with a solid release film, such as Teflon. A scheme of the defects is shown in Fig. 1 (b). Here, the skin thickness 


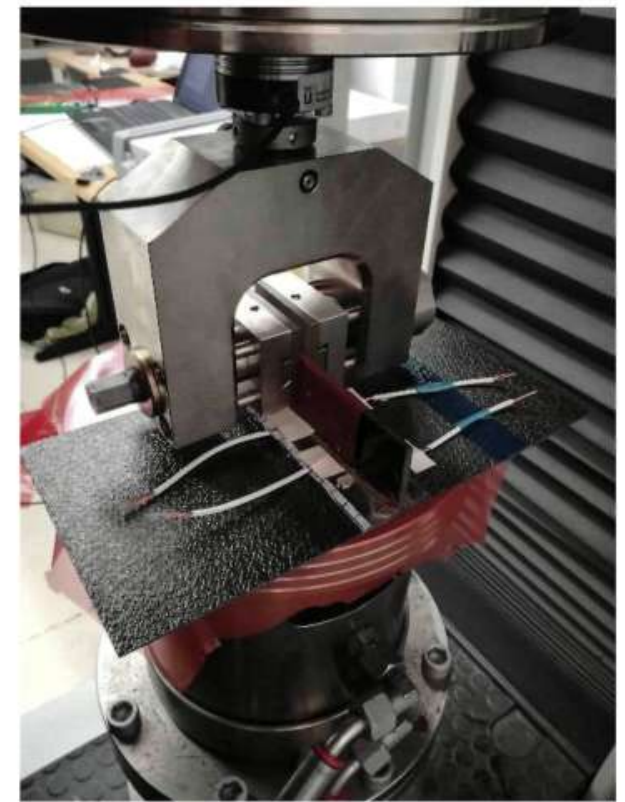

(a)

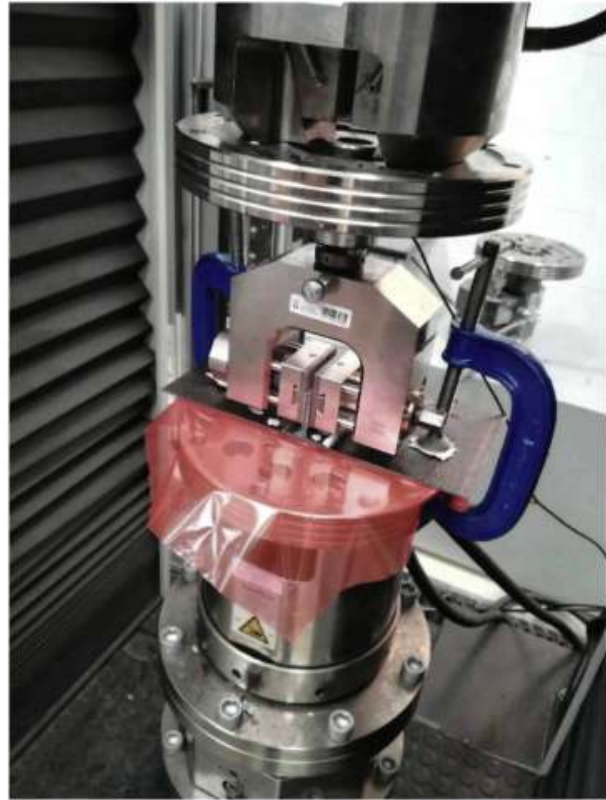

(b)

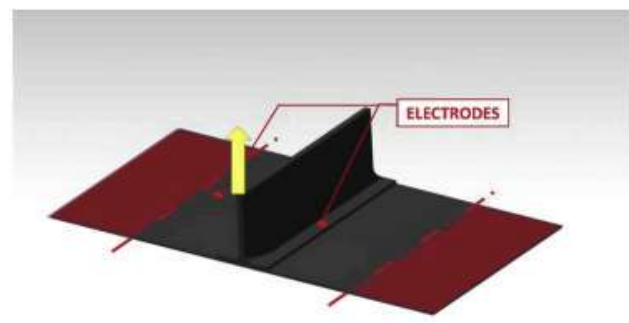

(c)

Fig. 2. Images of (a), (b) peeling test of skin-stringer sub-elements and (c) schematics of electrodes yellow arrow indicates the direction of the applied load and red areas the clamped regions. (For interpretation of the references to colour in this figure legend, the reader is referred to the Web version of this article.)

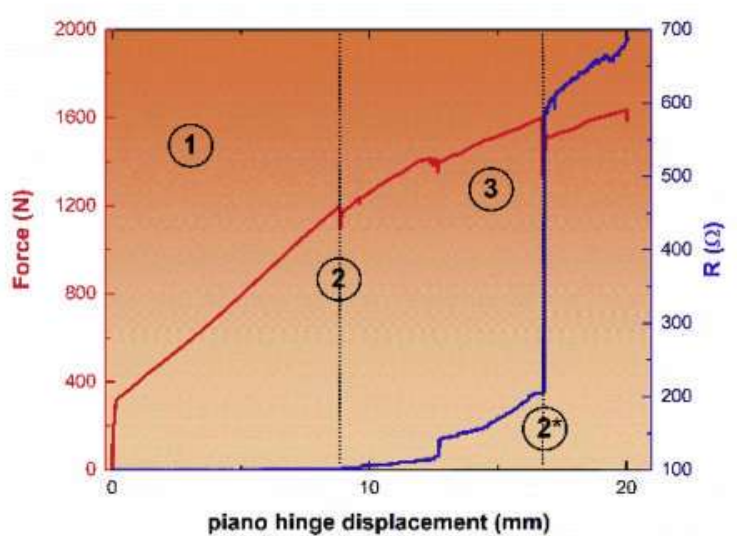

(a)

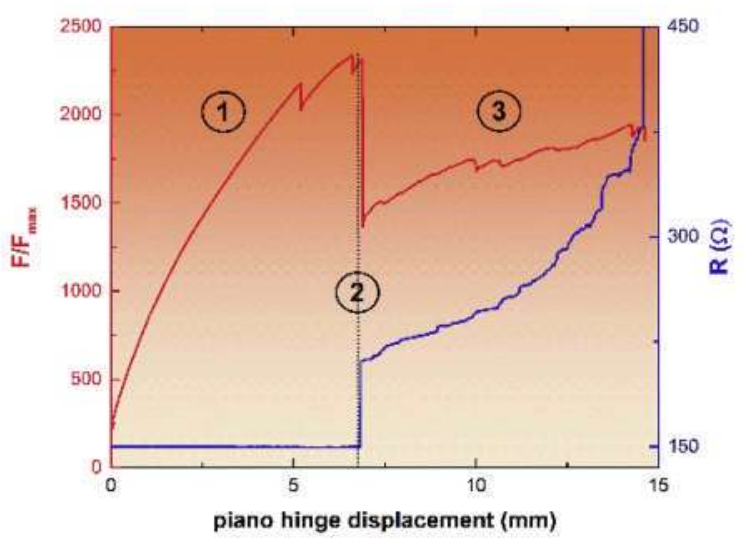

(b)

Fig. 3. Electromechanical response of the (a) co-bonded and (b) secondary bonded undamaged stiffened elements (red curve denotes the load and blue the electrical resistance variation). (For interpretation of the references to colour in this figure legend, the reader is referred to the Web version of this article.) 


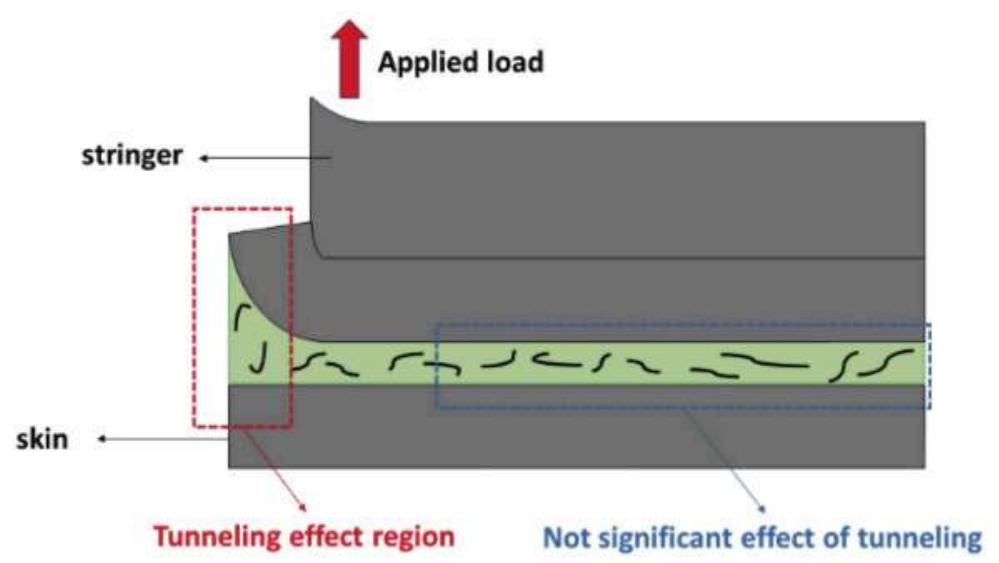

(a)

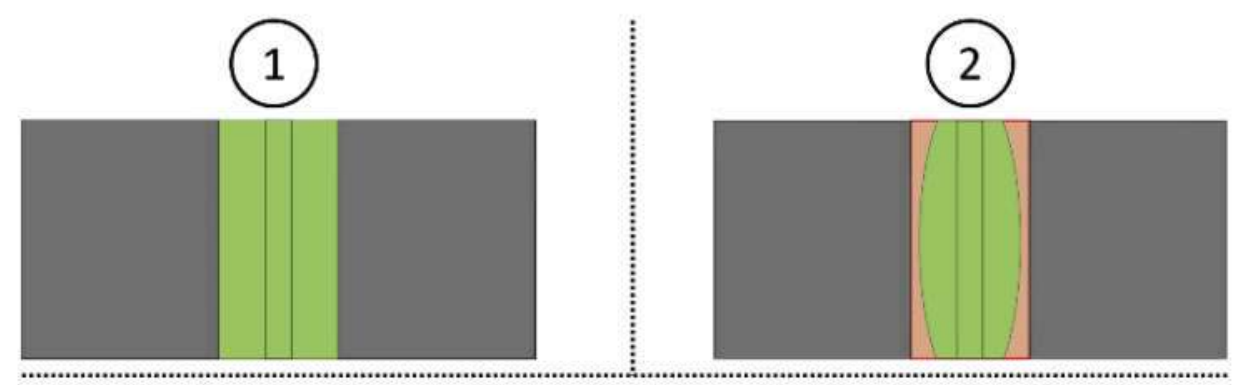

3

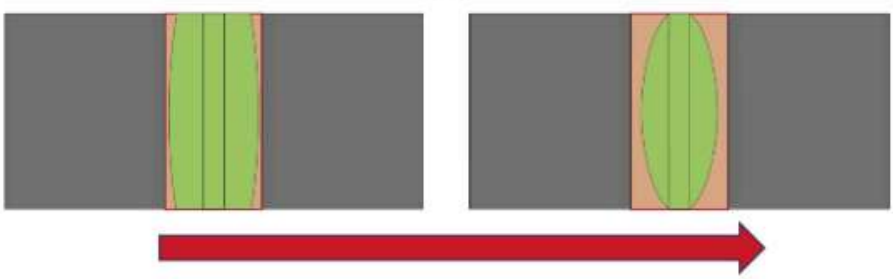

(b)

Fig. 4. Schematics of (a) deformation of adhesive joint from the side view of the specimen and (b) top view of adhesive joint showing the crack propagation (highlighted in red) in the stiffened element during peeling tests where 1 denotes the initial stage, 2 the first sudden propagation and 3 the crack propagation inside the joint. (For interpretation of the references to colour in this figure legend, the reader is referred to the Web version of this article.)

varies from $2 \mathrm{~mm}$ in the case of the secondary bonded specimens to 1.5 $\mathrm{mm}$ in the case of the co-bonded ones, due to manufacturing issues.

\subsection{Mechanical and structural health monitoring tests}

Both stiffened and standard specimens were subjected to peeling tests in a Zwick universal tensile machine as shown in Fig. 2 (a) and (b). Test rate was set as $3 \mathrm{~mm} / \mathrm{min}$ and the crack propagation was recorded by using a video camera.

During the peeling test, electrical response was monitored by means of electrical resistance measurements using an Agilent 34400A module hardware. Electrodes, whose disposition is shown in Fig. 2 (c), were made of copper wire sealed to the substrate using silver ink and covered with an adhesive layer to protect them from the environment.

\subsection{Microstructural analysis}

The analysis of the cross-section of the adhesive bond line was carried out by Scanning Electron Microscopy (SEM) using a S-340ON apparatus from Hitachi. To achieve this purpose, several sections of the co-bonded and secondary bonded skin-stringer joints were properly polished and gold-coated by sputtering for a good observation of the microstructure.

\section{Results and discussion}

In this section, a detailed electromechanical analysis of the skinstringer elements is carried out. Firstly, electromechanical behaviour of the co-bonded and secondary bonded specimens is shown and analysed by stating the main differences in their electrical and mechanical responses. Then, a microstructural analysis of cross-sections is carried 


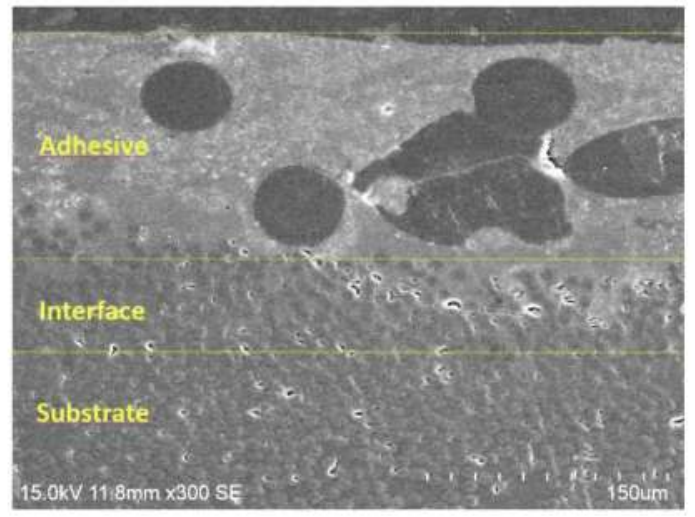

(a)

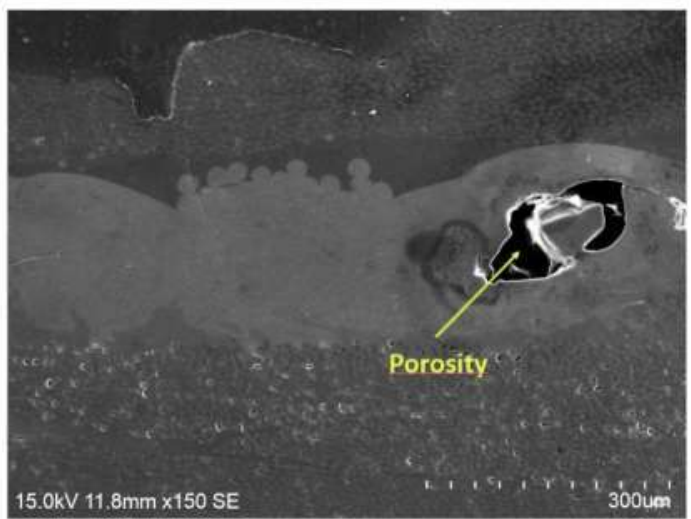

(c)

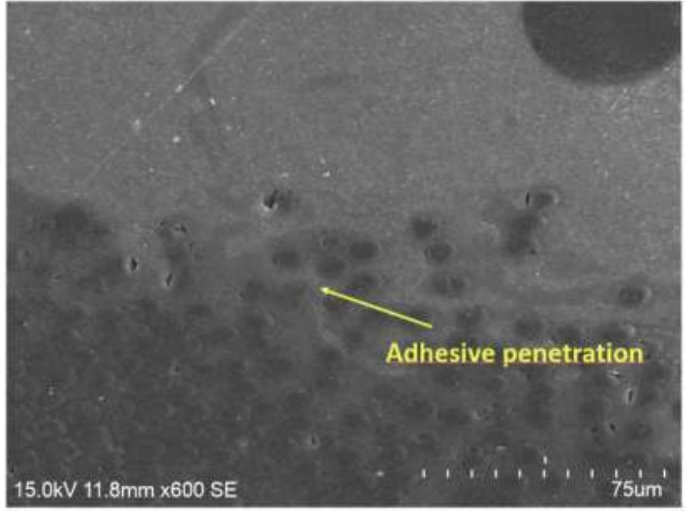

(b)

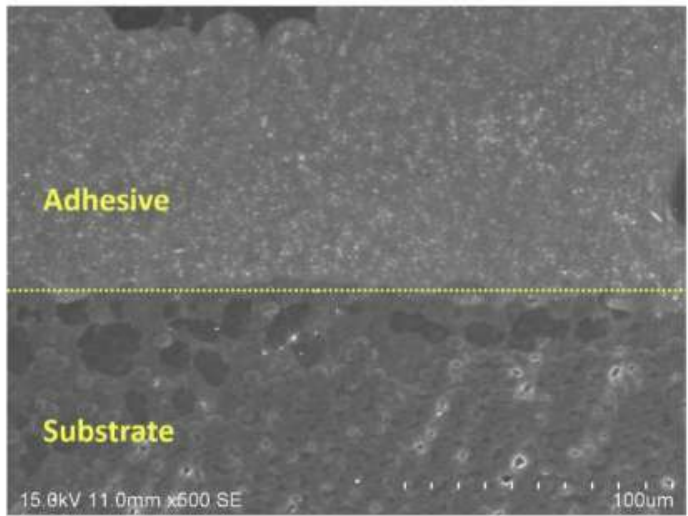

(d)

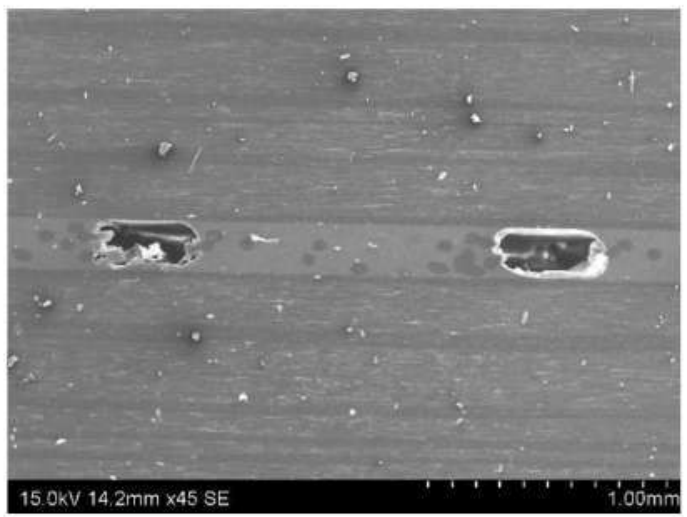

(e)

Fig. 5. SEM images of the transverse section of the adhesive joint for co-bonded joints showing (a) the substrate, interface and adhesive regions, (b) the adhesive penetration into the skin and (c) some localised porosity and secondary bonded joints showing (d) the interface adhesive-substrate and (e) the presence of voids.

out to identify the main interfacial mechanisms which participate in the bond failure. After that, an evaluation of the defects is performed, highlighting the differences observed in the two types of mechanical testing applied: mode-I standard ones and peeling on the skin-stringers where the influence of different types of artificial tests, as liquid or solid release agents, was deeply analysed. Finally, an analysis of the fracture surfaces is also performed.

\subsection{Electromechanical analysis of co-bonded and secondary bonded specimens}

Fig. 3 shows representative results of the electromechanical curves obtained during the peeling tests. As a general trend, the electrical resistance increases with applied load. This is in good agreement with previous studies for Mode-I specimens [32], where the breakage of electrical pathways induced by the crack propagation during the test, also leads to a sudden increase of electrical resistance. Here, it can be stated that the maximum applied load of the co-bonded specimens is 


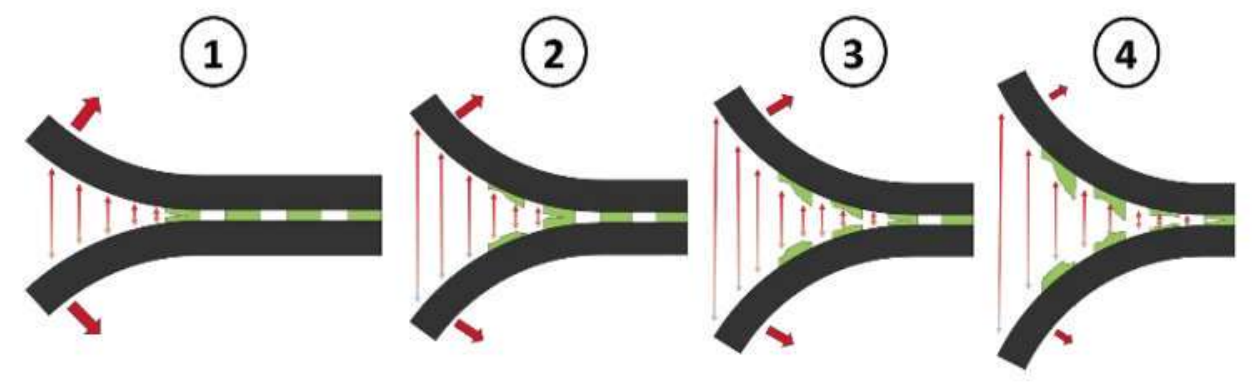

(a)

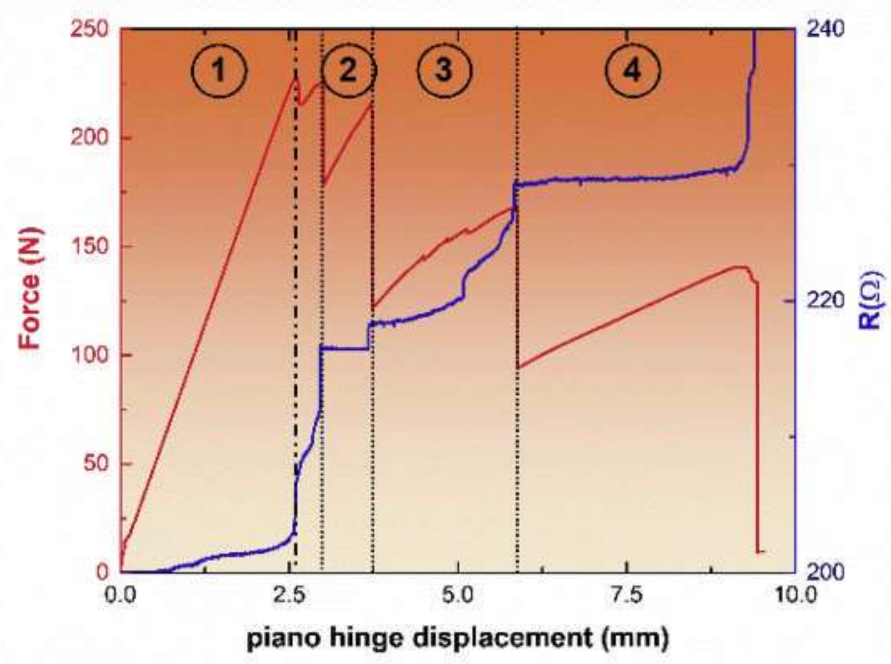

(b)

Fig. 6. (a) Schematics of crack propagation and (b), (c) electromechanical behaviour of damaged Mode-I specimen (where red curves denotes the load and blue the electrical resistance). (For interpretation of the references to colour in this figure legend, the reader is referred to the Web version of this article.)

lower than the secondary bonded. This is explained by the lower thickness of the skin, as previously stated, that affects the mechanical performance. For this reason, the analysis will be focussed on the electrical characterisation.

Furthermore, it is possible to distinguish different regions according to the crack propagation and electrical response of the sub-elements (marked as points 1, 2 and 3 respectively, in Fig. 3). The first one corresponds to the initial stage of the peeling tests. Here, the crack does not yet nucleate so the most prevalent effect is the adhesive deformation which induces an increase of distance between adjacent nanoparticles and, thus, an increase of electrical resistance associated to this effect. However, the effect is less significant than in tensile or single lap shear conditions, as observed in previous studies [32,34]. This is explained because only a small part of the joint is subjected to the maximum strain level, as shown in the schematics of Fig. 4.

The second region is correlated to a rapid crack propagation where a drop of mechanical strength is observed. In this case, there is a sudden increase of electrical resistance due to a rapid breakage of electrical pathways.

The third region is identified as a continuous crack propagation inside the joint. It is correlated to a softer increase of the electrical resistance due to a more uniform crack propagation, so the interruption of electrical pathways is less drastic.

This is the general situation of a typical electromechanical behaviour. However, when comparing the stiffened elements manufactured by co-bonding and by secondary bonding, some significant differences can be observed.

In the case of the co-bonded element, a soft increase of the electrical resistance is observed in the first stages of crack propagation, where it grows through the edges. Then, a sharp increase is observed, marked at point $2^{*}$ in Fig. 3 (a), and is correlated to a sudden drop in the mechanical response. When this sudden crack propagation occurs, it reaches the total length of the stringer. Finally, there is again a soft increase of the electrical resistance until final failure of the specimen.

However, in the case of secondary bonded elements, the initial crack propagation takes place in a very sudden way, corresponding to a sudden drop of the mechanical load (Fig. 3 (b)). This behaviour is explained by a sudden crack growth through the whole joint. As observed by the video camera during the test, there is a quick crack propagation following the stringer edge direction. Afterwards, the crack starts to grow towards the centre of the joint, due to the bending strains applied in the skin, as schematised in Fig. 4 (b). This propagation towards the centre is reflected in a soft increase of the electrical resistance, marked as point 3 in Fig. 3 (b). It is in good agreement with the mechanical response, where no sudden drops due to rapid crack propagation are observed.

The differences observed between both types of bonded elements are correlated to the way in which the stiffened elements are manufactured, 

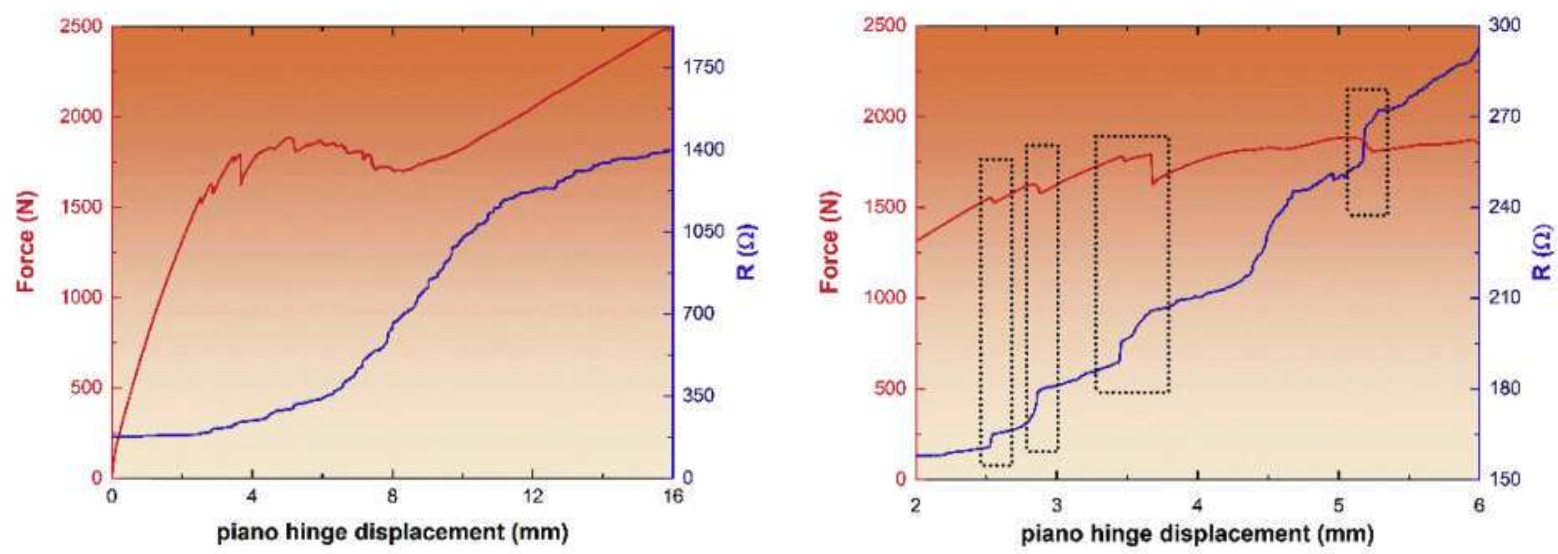

(a)
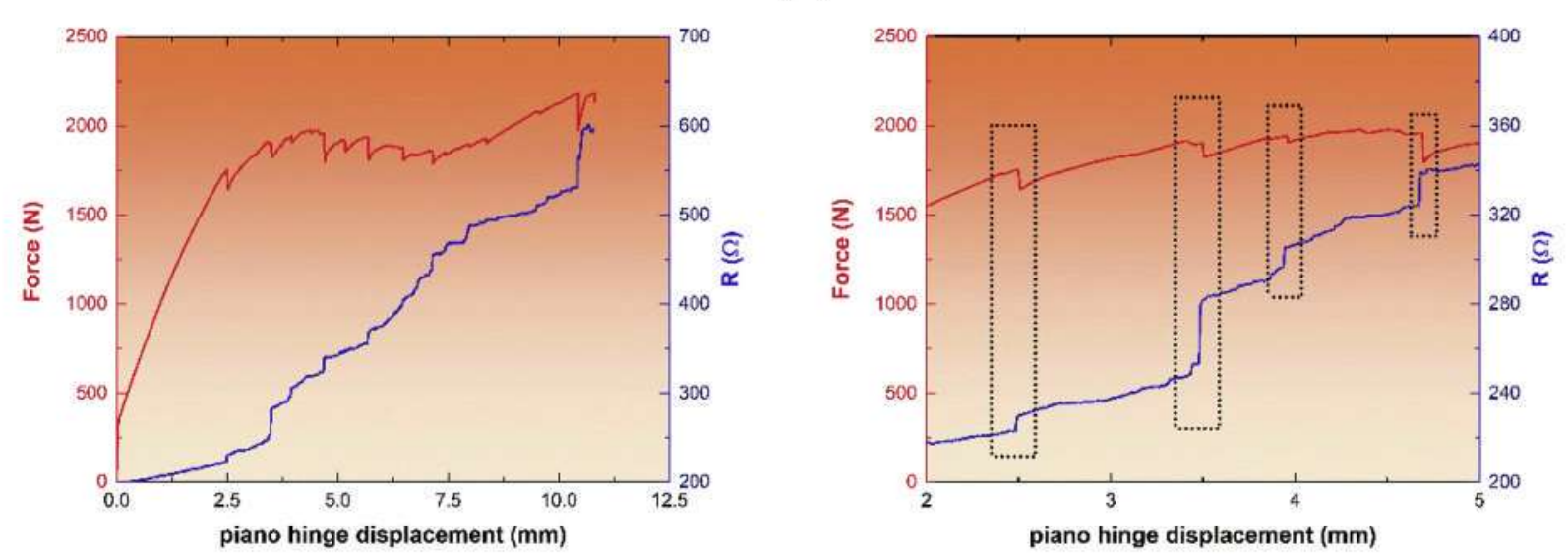

(b)

Fig. 7. Electromechanical and detailed response of stiffened elements with (a) liquid release agent and (b) Teflon insert. (NOTE: the figures on the right are just a zoomed plot of the most interesting detail of the global response (left)).

which has a significant influence on the adhesive properties of the joint. In this context and for a better explanation, a microstructural analysis of the joints is carried out.

\subsection{Microstructural analysis of co-bonded and secondary bonded elements}

Fig. 5 shows SEM microstructures of the polished cross-section of the adhesive joint between the skin and the stringer.

In the case of the co-bonded specimens, three regions can be distinguished: the CFRP substrate, the interface between the adhesive and substrate, and the adhesive itself (Fig. 5 (a)). This intermediate region is formed by the adhesive that flows during curing and penetrates into the uncured skin (Fig. 5 (b)). Here, a generally continuous adhesivesubstrate interface is observed through the whole joint. However, some localised porosity is observed in both the adhesive joint and the skin substrate (Fig. 5 (c)). This is explained by the lack of compaction induced by the manufacturing at vacuum conditions.

In the case of the secondary bonded specimen, the commented intermediate region is not found as both substrates have been previously cured (Fig. 5 (d)) so the adhesive does not penetrate into the skin. Therefore, the quality of the interface between substrate and adhesive is not as high as in the co-bonded elements. In addition to that, the presence of voids is more generalised (Fig. 5 (e)). This can be explained by the action of the weight of the T-stringer that could induce some overflow and, thus, a lack of adhesion, as has been previously observed in other studies $[32,33,35]$.

\subsection{Assessment of defects in adhesive joints}

The effect of damage in the mechanical and electrical response of the joint was also investigated. First, a Mode-I standard damaged specimen was tested. The artificial defects were induced by using a solid release film (Teflon). Fig. 6 shows the electromechanical response of the sample. Here, each sudden increase of electrical resistance is correlated to a drop of the mechanical load. This pattern was previously observed in stickslip propagation for Mode-I tests [32,36]. It is explained by the action of Teflon inserts that induce a total disruption of the electrical and mechanical pathways between the adhesive and the substrate. Therefore, the crack propagation is highly unstable as well as the electrical response.

A detailed analysis shows that electrical resistance slightly increases at the initial stages due to the adhesive deformation until the crack starts to propagate, where a drop of mechanical load is observed and, thus, the electrical resistance rapidly increases (marked as 1 on Fig. 6). Then, once the crack propagates through the first adhesive region, it finds the Teflon insert. Here, the mechanical load starts to increase until the crack again propagates through the next adhesive zone (marked as 2 on Fig. 6). This process takes place repeatedly through the different adhesive areas (marked as 3 and 4 on Fig. 6). Here, it is interesting to point out that the slight increase of electrical resistance between areas of sudden crack propagation are explained because of the effect of adhesive 


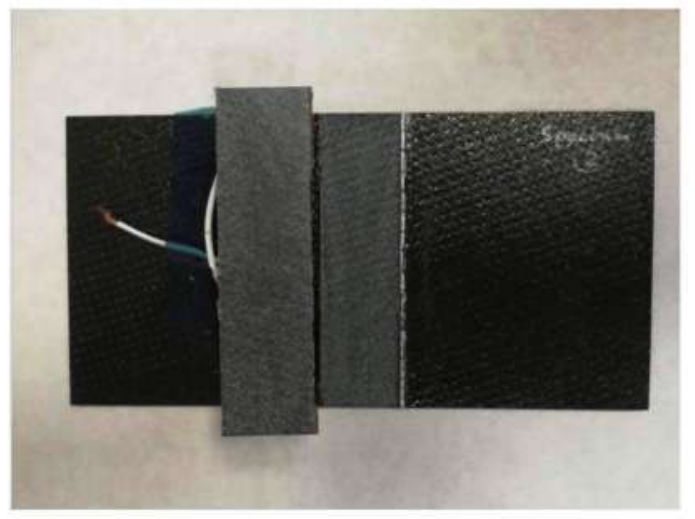

(a)

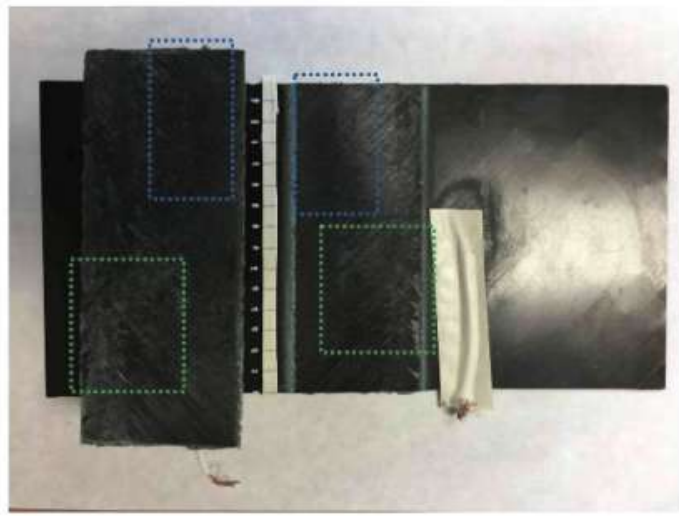

(c)

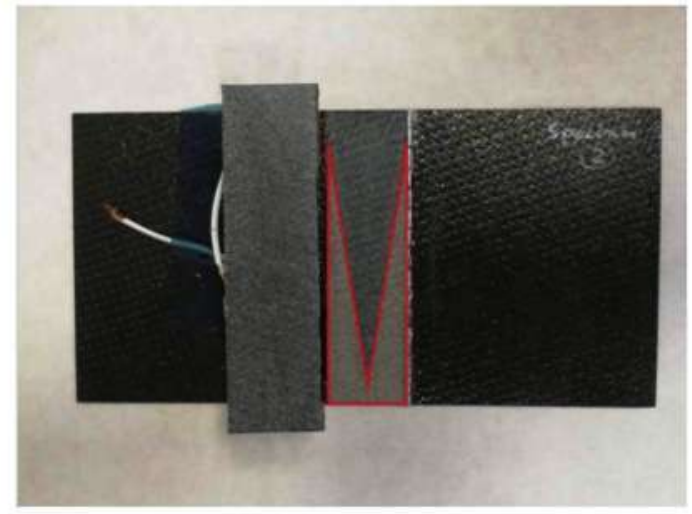

(b)

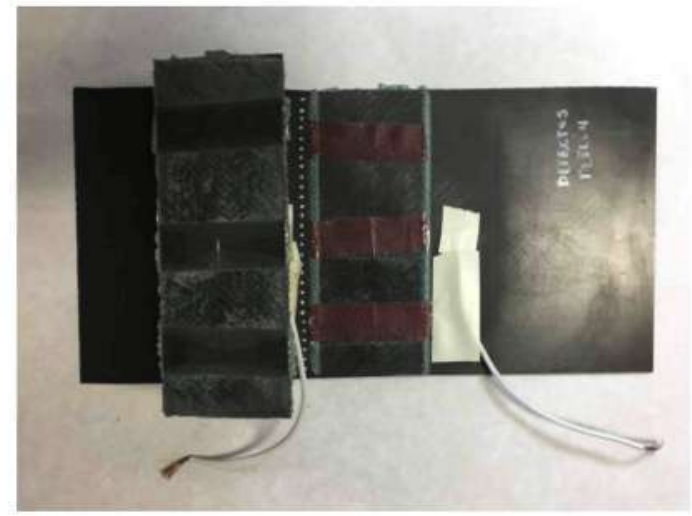

(d)

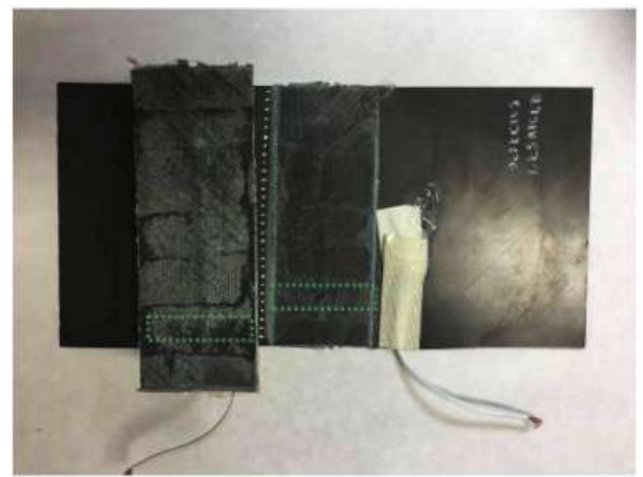

(e)

Fig. 8. Image of the fracture surface showing (a) the effect of co-bonding and (b) the pattern of the crack propagation (highlighted in red) and the effect of (c) secondary bonding and (d) Teflon and (e) liquid agent inserts. (For interpretation of the references to colour in this figure legend, the reader is referred to the Web version of this article.)

deformation, as explained before, which induces an increase of tunnelling resistance. Therefore, at first sight, there is a high agreement between the mechanical and electrical behaviour of the damaged normalised coupons.

In this regard, by using this information, Fig. 7 summarises the electromechanical behaviour of the damaged stiffened elements. Although at a first sight both liquid and solid release agents show a similar behaviour, some differences can also be observed.

The increase of the electrical resistance in the case of the liquid agent is softer than in the Teflon specimen. This can be explained attending to the quality of the interface and the way in which crack trends to propagate in both cases.

The liquid agent does not induce a complete discontinuity inside the adhesive joint. For this reason, the crack propagation does not take place in a sudden way, as in case of the Teflon insert where there is a total discontinuity between the adhesive and the substrate, as commented before. In addition, crack propagation takes places mainly in the weak interfaces induced by the liquid agent. This fact promotes a softer crack propagation as observed in other studies [36]. Then, the Teflon damaged specimens, which simulate joints with de-bonded areas, showed a 
sharper increase of the electrical resistance during the peeling test with several drops of the mechanical response. By analysing the curves in detail, an excellent agreement between electrical and mechanical response is observed, with each sharp increase of electrical resistance corresponding to a sudden drop of the mechanical response, as can be observed in the detailed graph of Fig. 7 (b).

Furthermore, it can be noticed that the electrical resistance variation at the initial stage is higher than in the undamaged specimens, where it was almost zero. This can be explained by the fact that artificial defects can induce an early nucleation of cracks during the first stages of the peeling tests, promoting a higher variation of the electrical response.

The analysis of the fracture surfaces reveals that the co-bonded specimens show a totally cohesive failure (Fig. 8 (a)) with the presence of some patterned areas corresponding to the main crack propagation mechanisms inside the joint (Fig. 8 (b)). However, in case of secondary bonding, there is a mixed failure mode with some areas showing a cohesive failure and even a delamination of the substrate (green and blue areas of Fig. 8 (c), respectively). By looking at the damaged specimens, a much higher discontinuity of the joint is observed when placing the Teflon inserts, where a total adhesive failure is observed in these areas (Fig. 8 (d)). On the other hand, liquid agent treated zones, which would simulate low strength bonded areas with intimate contact (kissing bond), induce softer discontinuities with the presence of cohesive regions inside the defect (Fig. 8 (e)). This is in total agreement with the electrical response, with a sharper behaviour in the case of the Teflon inserts due to the higher discontinuity of the electrical pathways.

\section{Conclusions}

The influence of the manufacturing process and defects has been analysed in standard coupons and skin-stringer elements by means of electrical monitoring of carbon nanotube doped adhesive films.

It was observed that the electrical resistance increases with crack propagation, making it possible to distinguish different regions: a first one with a slight increase due to tunnelling mechanisms when there is no crack nucleation; a second one characterised by a sharp increase due to rapid crack propagation, and a third one with a softer increase due to a more uniform crack propagation inside the joint. In this context, the manufacturing process has a significant effect on electromechanical behaviour. Joints manufactured by secondary bonding show a much sharper increase of electrical resistance in the first stages of the test because of a sudden crack propagation following the joint edges.

Furthermore, it is observed that artificial defects also have a deep impact on the electromechanical properties. Samples with Teflon film inserts, which simulated debonded areas, show a more unstable electrical response as crack propagation takes place, also in a more unstable way. On the other hand, samples with the liquid agent, which simulates a kissing bond, show a more uniform behaviour due to a more stable crack propagation inside the joint. In every case, each sudden mechanical drop corresponds to a sharp increase of electrical resistance, so the high potential and applicability of the proposed method is demonstrated by identifying different types of defects and crack propagation.

\section{Declaration of competing interest}

The authors have no affiliation with any organization with a direct or indirect financial interest in the subject matter discussed in the manuscript.

\section{CRediT authorship contribution statement}

Xoan F. Sánchez-Romate: Conceptualization, Methodology, Formal analysis, Writing - original draft. Rocío Moriche: Conceptualization, Methodology, Writing - review \& editing. Ángel Renato Pozo: Conceptualization, Methodology. Alberto Jiménez-Suárez:
Conceptualization, Writing - review \& editing. María Sánchez: Writing - review \& editing. Alfredo Güemes: Supervision, Writing - review \& editing. Alejandro Ureña: Funding acquisition, Writing - review \& editing.

\section{Acknowledgements}

This work was supported by the Ministerio de Economía y Competitividad of the Spanish Government [Project MAT2016-78825-C2-1$\mathrm{R}$ ] and Comunidad de Madrid Government (Project ADITIMAT-CM (S2018/NMT-4411)).

\section{Appendix A. Supplementary data}

Supplementary data to this article can be found online at https://doi. org/10.1016/j.compscitech.2020.108147.

\section{References}

[1] R. Hussey, J. Wilson, Structural Adhesives: Directory and Databook, Springer Science \& Business Media, 1996.

[2] N. Saleema, D.K. Sarkar, R.W. Paynter, D. Gallant, M. Eskandarian, A simple surface treatment and characterization of AA 6061 aluminum alloy surface for adhesive bonding applications, Appl. Surf, Sci, 261 (2012) 742-748.

[3] L. Sorrentino, W. Polini, C. Bellini, G. Parodo, Surface treatment of CFRP: influence on single lap joint performances, Int. J. Adhesion Adhes. 85 (2018) 225-233.

[4] X. Zhu, Y. Li, J. Ni, X. Lai, Curing-induced debonding and its influence on strength of adhesively bonded joints of dissimilar materials, Journal of Manufacturing Science and Engineering-Transactions of the Asme 138 (6) (2016), 061005.

[5] F. Heidarpour, M. Farahani, P. Ghabezi, Experimental investigation of the effects of adhesive defects on the single lap joint strength, Int. J. Adhesion Adhes. 80 (2018) $128-132$.

[6] A. Bernasconi, M. Carboni, L. Comolli, Monitoring of fatigue crack growth in composite adhesively bonded joints using fiber Bragg gratings, Procedia Engineering 10 (2011) 207-212.

[7] F. Habib, M. Martinez, A. Artemev, M. Brothers, Structural health monitoring of bonded composite repairs : a critical comparison between ultrasonic Lamb wave approach and surface mounted crack sensor approach, Compos. B Eng. 47 (2013) 26-34.

[8] A. Crawford, M.G. Droubi, N.H. Faisal, Analysis of acoustic emission propagation in metal-to-metal adhesively bonded joints, J. Nondestr. Eval. 37 (2) (2018) 33.

[9] H. Duflo, B. Morvan, J. Izbicki, Interaction of Lamb waves on bonded composite plates with defects, Compos. Struct. 79 (2) (2007) 229-233.

[10] F.R. Flor, R. de Medeiros, V. Tita, Numerical and experimental damage identification in metal-composite bonded joint, J. Adhes. $91(10-11)(2015)$ $863-882$.

[11] W.S. Na, Low cost technique for detecting adhesive debonding damage of glass epoxy composite plate using an impedance based non-destructive testing method, Compos. Struct. 189 (2018) 99-106.

[12] R.S. Ruoff, D.C. Lorents, Mechanical and thermal properties of carbon nanotubes, Carbon 33 (7) (1995) 925-930.

[13] M.F.L. De Volder, S.H. Tawfick, R.H. Baughman, A.J. Hart, Carbon nanotubes: present and future commercial applications, Science 339 (6119) (2013) 535-539.

[14] J. Li, M. Ötling, Conductivity scaling in supercritical percolation of nanoparticles-not a power law, Nanoscale 7 (8) (2015) 3424-3428.

[15] R. Nadiv, R.M.F. Fernandes, G. Ochbaum, J. Dai, M. Buzaglo, M. Varenik, R. Biton, I. Furo, O. Regev, Polymer nanocomposites: insights on rheology, percolation and molecular mobility, Polymer 153 (2018) 52-60.

[16] K. Tserpes, C. Kora, A multi-scale modeling approach for simulating crack sensing in polymer fibrous composites using electrically conductive carbon nanotube networks. Part I: micro-scale analysis, Comput. Mater. Sci. 154 (2018) 530-537.

[17] Hu N. Alamusi, H. Fukunaga, S. Atobe, X. Liu, J. Li, Piezoresistive strain sensors made from carbon nanotubes based polymer nanocomposites, Sensors 11 (11) (2011) 10691-10723.

[18] A. Sanli, A. Benchirouf, C. Mueller, O. Kanoun, Piezoresistive performance characterization of strain sensitive multi-walled carbon nanotube-epoxy nanocomposites, Sens. Actuators, A 254 (2017) 61-68.

[19] S.K. Reddy, S. Kumar, K.M. Varadarajan, P.R. Marpu, T.K. Gupta, M. Choosti, Strain and damage-sensing performance of biocompatible smart CNT/UHMWPE nanocomposites, Mater. Sci. Eng. C 92 (2018) 957-968.

[20] X.F. Sánchez-Romate, J. Artigas, A. Jiménez-Suárez, M. Sánchez, A. Güiemes, A. Urena, Critical parameters of carbon nanotube reinforced composites for structural health monitoring applications: empirical results versus theoretical predictions, Compos, Sci. Technol. 171 (2019) 44-53.

[21] S. Aziz, S. Chang, Smart-fabric sensor composed of single walled carbon nanotubes containing binary polymer composites for health monitoring, Compos, Sci. Technol. 163 (2018) 1-9.

[22] Z. Chen, X. Liu, S. Wang, X. Zhang H. Luo, A bioinspired multilayer assembled microcrack architecture nanocomposite for highly sensitive strain sensing, Compos. Sci. Technol. 164 (2018) 51-58. 
[23] L. Shen, L. Liu, W. Wang, Y. Zhou, In situ self-sensing of delamination initiation and growth in multi-directional laminates using carbon nanotube interleaves, Compos. Sci. Technol. 167 (2018) 141-147.

[24] T. Augustin, J. Karsten, B. Kötter, B. Fiedler, Health monitoring of scarfed CFRP joints under cyclic loading via electrical resistance measurements using carbon nanotube modified adhesive films, Compos. Appl. Sci. Manuf. 105 (2018) 150-155.

[25] O. Sam-Daliri, L. Faller, M. Farahani, A. Roshanghias, A. Araee, M. Baniassadi, H. Oberlercher, H. Zangl, Impedance analysis for condition monitoring of single lap CNT-epoxy adhesive joint, Int. J. Adhesion Adhes, 88 (2019) 59-65.

[26] A.S. Lim, Z.R. Melrose, E.T. Thostenson, T. Chou, Damage sensing of adhesivelybonded hybrid composite/steel joints using carbon nanotubes, Compos. Sci. Technol. 71 (9) (2011) 1183-1189.

[27] M. Kang, J. Choi, J. Kweon, Fatigue life evaluation and crack detection of the adhesive joint with carbon nanotubes, Compos. Struct. 108 (2014) 417-422.

[28] M.R. Gude, S.G. Prolongo, T, Gomez del Rio, A. Urena, Mode-I adhesive fracture energy of carbon fibre composite joints with nanoreinforced epoxy adhesives, Int J. Adhesion Adhes. 31 (7) (2011) 695-703.

[29] I.M. Wernik, S.A. Meguid, On the mechanical characterization of carbon nanotube reinforced epoxy adhesives, Mater. Des. 59 (2014) 19-32.

[30] I.A. Akpinar, K. Guiltekin, S. Akpinar, H. Akbulut, A. Ozel, Research on strength of nanocomposite adhesively bonded composite joints, Compos. B Eng. 126 (Supplement C) (2017) 143-152,
[31] C. Garcia, M. Sanchez, A. Urena, J. Rams, Carbon nanotubes for assessing the structural integrity of composite bonded joints with film adhesives, Structural Health Monitoring 2015: System Reliability for Verification and Implementation 1 (2) (2015) 2169-2176.

[32] X.X. Fernández Sảnchez-Romate, J. Molinero, A. Jiménez-Suárez, M. Sánchez, A. Güemes, A. Ureña, Carbon nanotube doped adhesive films for detecting crack propagation on bonded joints: a deeper understanding of anomalous behaviors, ACS Appl. Mater. Interfaces 9 (49) (2017) 43267-43274.

[33] X.F. Sanchez-Romate, A. Jiménez-Suárez, J. Molinero, M. Sánchez, A. Güemes, A. Urena, Development of bonded joints using novel CNT doped adhesive films: mechanical and electrical properties, Int. J. Adhesion Adhes. 86 (2018) 98-104.

[34] X.F. Sánchez-Romate, L. Baena, A. Jiménez-Suárez, M. Sánchez, A. Güemes, A. Urena, Exploring the mechanical and sensing capabilities of multi-material bonded joints with carbon nanotube-doped adhesive films, Compos. Struct. 229 (2019), 111477.

[35] X.F. Sánchez-Romate, R. Moriche, A. Jiménez-Suárez, M. Sánchez, A. Güemes, A. Ureña, An approach using highly sensitive carbon nanotube adhesive films for crack growth detection under flexural load in composite structures, Compos. Struct. 224 (2019), 111087.

[36] T. Takeda, F. Narita, Fracture behavior and crack sensing capability of bonded carbon fiber composite joints with carbon nanotube-based polymer adhesive layer under Mode I loading, Compos. Sci. Technol. 146 (2017) 26-33. 